\title{
Eating Disorders and the Use of Cognitive Enhancers and Psychostimulants Among University Students: A Cross-Sectional Study
}

\author{
Basma Damiri (1D' \\ Omar A Safarini ${ }^{2}$ \\ Zaher Nazzal (1D ${ }^{2}$ \\ Ahmad Abuhassan ${ }^{3}$ \\ Ahmad Farhoud ${ }^{2}$ \\ Nesma Ghanim ${ }^{4}$ \\ Rayyan Al Ali ${ }^{5}$ \\ Mirvat Suhail $^{2}$ \\ Mohammad Qino ${ }^{2}$ \\ Mohammad Zamareh ${ }^{2}$ \\ Ammar Thabaleh ${ }^{2}$ \\ Jihad Zahran ${ }^{2}$
}

'Medicine \& Health Sciences Faculty, Drug, and Toxicology Division, An-Najah National University, Nablus, 00970 , Palestine; ${ }^{2}$ Medicine \& Health Sciences Faculty, Department of Medicine, AnNajah National University, Nablus, 00970, Palestine; ${ }^{3} \mathrm{An}-\mathrm{Najah}$ National University Hospital, Department of Neurology, AnNajah National University, Nablus, 00970, Palestine; ${ }^{4}$ Medicine \& Health Sciences Faculty, Department of Public Health, AnNajah National University, Nablus, 00970, Palestine; ${ }^{5}$ Medicine \& Health Sciences Faculty, Forensic Medicine Institute, AnNajah National University, Nablus, 00970, Palestine
Correspondence: Basma Damiri Faculty of Medicine and Health Sciences, Drug and Toxicology Division, An-Najah National University, PO Box 7, Nablus, 00970, Palestine

Tel +972592234270

Email bdamiri@najah.edu
Background: University students, who are at risk of eating disorders (ED), are more liable to use cognitive enhancers and psychostimulants to improve their cognitive functions and lose weight. ED in Palestinian male students is a neglected health issue. We aimed to investigate the prevalence and the association between ED and cognitive enhancers, and psychostimulants use among An-Najah National University students (ANNU).

Methods: In a cross-sectional study conducted in 2020 at ANNU, 1047 students completed anonymous surveys for cognitive enhancers practice, the Eating Attitude Test-26 (EAT-26), Sick, Control, One, Fat and FOOD (SCOFF) screening tests.

Results: The prevalence of ED among ANNU students was $21.2 \%$ based on EAT-26 $(17.1 \%$ in males, $23.8 \%$ in females) and $31.5 \%$ based on SCOFF $(24.0 \%$ in males, $36.3 \%$ in females). The binary logistic regression revealed that students at risk to have ED were waterpipe smokers (OR: 1.471, p-value 0.047), especially males, while students who were less likely to have ED were males (OR: 0.341, p-value $<0.001$ ), coffee users (OR: 0.581, p-value 0.014 ) and chocolate users (OR: 0.530, p-value 0.041) than nonusers. Moreover, the risk of ED increased with increasing body mass index ( $p$-value $<0.01$ ). Clinical medical students showed the lowest prevalence $(11.1 \%)$ compared to preclinical $(22.5 \%)$, health sciences (23.7\%), and non-medical students (20.9\%) (p-value 0.059).

Conclusion: Our findings highlight water-pipe smoking as a significant health problem in males with $\mathrm{ED}$, which may require unique treatment and prevention strategies. Moreover, coffee and chocolate consumption were associated with decreased risk of ED, only among males. The gender-gap in ED prevalence was very narrow compared to international results. These results prompt the need to focus on both genders in future studies instead of females. They also suggest the urgent need to address ED among Palestinian university students by educating students about mental health, identifying high-risk students, and offering easily accessible psychological help.

Keywords: cognitive enhancers, psychostimulants, eating disorders, tobacco smoking, water-pipe smoking

\section{Plain Language Summary}

Eating disorders have been widely considered one of the most serious and debilitating psychiatric illnesses. Anorexia nervosa is a psychological eating disorder characterized by an obsessive desire to lose weight, food restriction, and excessive fear of gaining weight. It has the highest mortality rate among mental illnesses. It affects mostly young females and usually has its onset in adolescence. Bulimia nervosa is characterized by binge eating episodes followed by compensatory behaviors, and it is usually associated with comorbid 
psychopathology. University students, who are at risk of ED, reported concerns interfering with their academic performance. Therefore, they can use cognitive enhancers and psychostimulants to improve their cognitive functions and lose weight. ED in Palestinian male students is a neglected health issue. We aimed to investigate the prevalence and the association between ED and cognitive enhancers and psychostimulants use among An-Najah National University students (ANNU). Our results indicate that ED among Palestinian university students is more common than their international counterparts but compatible with some regional results. Moreover, males with higher body mass index and those who smoke water-pipe were at higher risk of having ED. Surprisingly, male students who consumed coffee had a lower risk of having ED than non-consumers and females. This is also the case for chocolate consumption. Therefore, there is a need to change the current stereotypical image of ED as a disease of females only, possibly causing ED to be under-diagnosed and under-treated in men.

\section{Introduction}

Eating disorders (ED) have been widely considered one of the most serious and debilitating psychiatric illnesses. ${ }^{1,2}$ They constitute a growing health problem with multifactorial etiology affecting adolescents and young adults worldwide, including Arabs. ${ }^{3-5}$ The available data regarding ED suggest multifactorial causation, including biological, familial, cultural, and environmental factors. ${ }^{6-8}$ Other suggested risk factors include female gender, age, weight, menarche age, social norms, academic performance, and peer pressure. ${ }^{5,9,10}$ With the growing influence of media and the expansion of perfectionism, university students are under more pressure to meet the perfect image. ${ }^{10-12}$ This ultimately leads to an increased incidence of psychological distress and ED among them, especially among medical students. ${ }^{13-16}$

University students, who were at risk for ED, reported concerns interfering with their academic performance. ${ }^{17}$ Therefore, they are liable to use cognitive enhancers and psychostimulants such as tobacco, coffee, and energy drinks to improve physical and cognitive functions. ${ }^{18}$ Tobacco smoking, cigarettes, and water-pipe are used for weight control as it has been shown to increase resting metabolic rate. ${ }^{19-23}$ Consuming excessive amounts of caffeine-containing fluids such as energy drinks may also serve as a weight control method to mask hunger, aid in purging behavior, or increase energy level. ${ }^{24}$ It was documented that caffeine consumption is associated with weight loss attempts and unhealthy eating behavior. ${ }^{24-26}$ Patients with eating disorders (anorexia nervosa and bulimia nervosa) believe that consuming large quantities of caffeine drinks will increase metabolic rate and decrease appetite. ${ }^{25}$ Hence, much attention should be paid to the high smoking prevalence and caffeine consumption in patients with ED. Moreover, chocolate has been previously linked to eating disorders, where chocolate craving has been linked to eating disturbances. For example, compulsive chocolate eater scored higher on measures of drive for thinness, body dissatisfaction, and bulimia, with a third of them, met the criteria of binge eating disorders. $^{27,28}$

Tobacco smoking and energy drinks have become nearly ubiquitous on university campuses in the West Bank, especially among males. ${ }^{18,29-37}$ The comorbidity of eating disordered behaviors and attitudes and cognitive enhancers and psychostimulants among Palestinian university students has not been considered in previous studies. $^{10,38}$ Little is known about ED behaviors and attitudes among Palestinian students. To our best knowledge, all previous national studies focused on determining the prevalence among only females. ${ }^{10,39}$ The prevalence among female students ranged from $28.6 \%$ to $36.9 \% .^{10,38}$ In Middle Eastern cultures, families tend to pressure girls and young women to eat, but also expect them to maintain an appropriate body weight and body size for marriage. ${ }^{11,12}$ These societal pressures may contribute to eating disordered behaviors in young female students at a critical age. ${ }^{11,12}$ The current stereotypical image of ED as a disease of females only causes ED to be under-diagnosed and under-treated in men. ${ }^{40,41}$ Furthermore, the stigma towards individuals with ED makes male patients feel ashamed and isolated. ${ }^{41}$ Males are less likely to seek psychological consultation than females. $^{42,43}$ Unfortunately, this delay in diagnosis could devastate psychiatric morbidity, leading to lifethreatening conditions. ${ }^{44}$ We aimed to investigate these gaps in the literature and explore the different factors associated with ED among university students at AnNajah National University (ANNU). This study is a part of ongoing research that investigates and assesses tobacco smoking, caffeine, energy drinks, and other substance use and comorbidity with different disorders. This study will help to better understand ED among Palestinian society, leading to earlier diagnosis and better outcomes, earlier interventions and treatments regarding ED, and providing recommendations to direct future research and treatment programs. 


\section{Materials and Methods}

\section{Study Design, Setting, and Sampling Technique}

A cross-sectional study was conducted among students at ANNU, a major university in the West Bank, Palestine. Students from ANNU were invited to participate in this study in the fall semester of 2020. Male to female ratio of 1:1 was implied. Then students were stratified according to their curricular year. Finally, proportional classification regarding the students' academic field was done $(50 \%$ medical and health sciences students and 50\% nonmedical students). Students who have hypo- or hyperthyroidism, Cushing syndrome, and epilepsy were excluded.

\section{Study Tool and Validity}

Paper-based surveys were used to achieve the research objectives. The Eating Attitude Test-26 (EAT-26) is a selfadministered questionnaire widely used to screen eating disorders. $^{45}$ It consists of 26 questions for which scoring is done on a 6-points scale from always to never. The EAT26 has been reproduced with permission Garner et al (1982). ${ }^{45}$ Sick, Control, One, Fat, and FOOD (SCOFF) is another valid self-administered questionnaire widely used also for screening for eating disorders. ${ }^{46}$ It consists of 5 questions; scoring is done on a 2-point scale, Yes or No. ${ }^{47}$ The Arabic-SCOFF questionnaire was accurate and reliable for the early detection of eating disorders. ${ }^{4}$ Arabic EAT-26 was also highly sensitive and specific for the early detection of eating disorders. ${ }^{4,48}$ The cutoff point of EAT-26 is 20; a score of 20 or more is considered at high risk of disordered eating attitudes and needs further investigation. ${ }^{45}$ In SCOFF, the cutoff point is 2; a score of 2 or more indicates a likely diagnosis of $\mathrm{AN}$ or $\mathrm{BN} .{ }^{46}$ The questionnaire used to assess cognitive enhancers and psychostimulants practice and pattern was used in a previous publication. ${ }^{32}$ It includes the history of consumption of these substances, the frequency of use, and the motives of these substances' use. A current user of a particular substance in this study is a student who has used this substance at least during the past 30 days. Energy drinks included imported and locally manufactured products available for purchase in Palestine at the time of the study. Anthropometric measurement, weight, and height were measured according to previous research. ${ }^{49}$ Briefly, weight, height, and waist were measured while the participants wearing light clothing and no shoes. Weight was determined to the nearest $0.1 \mathrm{~kg}$ and was measured using an electronic device. Height was determined to the nearest $0.1 \mathrm{~cm}$ and was measured using a wall meter. Body Mass Index (BMI) was calculated using this equation: weight $(\mathrm{kg})$ divided by the square of the height $\left(\mathrm{m}^{2}\right)$. The participants were classified into BMI categories: Underweight (BMI $<18.5)$, normal weight (BMI 18.5-24.9), overweight (BMI 25-29.9), and obese $\mathrm{BMI} \geq 30 .^{50}$

\section{Ethical Considerations}

The Ethics Committee of the Institutional Review Board "IRB" at An-Najah National University (ANNU) was obtained to confirm that the study matches the ethical research protocols. Written informed consent was obtained from each student prior to participation. An anonymous questionnaire was used. Participants had the ability to assent to or dissent from participation. Investigators from both genders were chosen to conduct this research as taking some data such as weight and height could be embarrassing for the participants if the investigator was from the opposite gender. Participants were assured that all of the data collected would only be kept confidential and only accessible for the investigators and research purposes. The questionnaires were kept in a secure place, and serial numbers were used to refer to participants during analysis and presenting data. The EAT-26 has been reproduced with permission Garner et al (1982). ${ }^{45}$

\section{Data Analysis}

All analyses were performed using IBM SPSS Statistics for Mac, version 22 (IBM Corp., Armonk, NY, USA). Standard univariate statistics were used to characterize the sample. Descriptive statistics were calculated for the point-prevalence of reported eating disorders and substance use. Differences in the means between groups were assessed using the independent samples $t$-test and analysis of variances (ANOVA). Associations between different general characteristics and outcomes were assessed using the Pearson's chi-square test and Fisher's Exact test. Binary logistic regression analysis was conducted to evaluate the relative risk by generating the odds ratios (OR) and 95\% confidence intervals (CI) for risk factors. A p-value of less than 0.05 was considered statistically significant.

\section{Results \\ Sociodemographic and Lifestyle Characteristics of the Students}

The total number of the distributed questionnaires was 1470 . The retained questionnaires were 1158 with a response rate 
of $78.9 \%$. Forty-three $(2.9 \%)$ students withdrew from the study, and 68 questionnaires $(4.6 \%)$ with incomplete information about EAT-26 or SCOFF tests were discarded. The final number of participants was 1047 (38.7\% males, 61.3\% females). The majority of the students $(98.2 \%)$ were singles and without work (87.7\%). Around 23.2\% were medical students, $32.0 \%$ were from health sciences, and $44.8 \%$ were non-medical academic fields. Twenty-eight percent of the students were overweight or obese (Table 1). Only $1.1 \%$ of the students were not using any substance, while $84 \%$ were using at least three substances, with a significant increase among males $(87.2 \%)$ compared to females $(82.1 \%)$ ( $p$-value 0.005$)$. The prevalence of substance use among the students was as the following: $41.1 \%$ for energy drinks, $17.6 \%$ for cigarette smoking, $30.1 \%$ for water-pipe smoking, $4.7 \%$ for e-cigarette smoking, $85.7 \%$ for coffee consumption, $84.4 \%$ for tea consumption, and $92.6 \%$ for chocolate consumption. There was a significant increase in the prevalence of cigarette, water-pipe, and electrical cigarette (e-cigarette) smoking among males compared to females ( $p$-value $<0.001$ ). There was significant increase in chocolate consumption among females $(96.7 \%)$ compared to males $(85.9 \%)$ ( $p$-value <0.001). No differences between males and females in coffee (p-value 0.999) and tea consumption (p-value 0.608 ) were observedpract (Table 1).

Supplementary Table 1 describes the pattern and the initiation age of the substances used. The most daily practiced substances were cigarettes (58.9\%), coffee $(50.6 \%)$, and tea $(45.2 \%)$. Chocolate $(35.2 \%)$, water-pipe $(23.6 \%)$, and e-cigarettes $(17.5 \%)$ were the least substances used. The initiation ages for the substances were 16.5 years for water-pipe in males and 17.7 years in females ( $p$-value 0.001), for energy drinks consumption (15.7 years in males and 16.7 in females, p-value <0.001), and coffee consumption (14.5 years in males and 15.1 in females, p-value 0.049) (Supplementary Table 1). Genderbased differences in the motivations of substance practice are described in Supplementary Table 2. To lose weight, $7.1 \%$ of the students smoked cigarettes, 5.4\% smoked water-pipe, and $6.0 \%$ smoked e-cigarettes. To less extent, $3.3 \%$ of the students consumed energy drinks, and $7.0 \%$ consumed coffee to lose weight.

\section{Prevalence of ED-Based in EAT-26 Score and SCOFF Score}

Based on EAT-26, the prevalence of ED was $21.2 \%(95 \%$ CI: $18.8-23.8 \%)$ and was higher among females $(23.8 \%)$ than males $(17.1 \%)$, (p-value 0.01). The prevalence increased with the increase in weight category (p-value $<0.001)$. The prevalence was higher in noncoffee users (28.7\%) than users (19.9\%) (p-value 0.015). Clinical medical students showed the lowest prevalence (11.1\%) compared to preclinical students $(22.5 \%)$, health sciences (23.7\%) students, and non-medical academic students $(20.9 \%)$ with no significant differences ( $p$-value 0.059). No significant differences were also determined between urban $(22.4 \%)$, rural $(20.1 \%)$, and refugee students $(16.0 \%)$, between singles $(21.2 \%)$ and none-singles $(21.1 \%)$ (p-value 0.625$)$, and between different academic years ( $p$-value 0.131). Based on the SCOFF scale, the prevalence of ED was $31.5 \%$ (95\% CI: $28.8-34.5 \%)$ with significant increase among females $(36.3 \%)$ compared to males $(24.0 \%)$ (p-value $<0.001)$ (Table 2$)$.

\section{Univariate Analysis for the Association Between Substance Use and EAT-26 Based on Gender}

Water-pipe smoking was associated with a higher risk of ED based on EAT-26 screening test among males (OR: 1.71, 95\% CI: 1.10-2.62, p-value 0.015). The risk of ED decreased among males who consumed coffee (OR: 0.475, 95\% CI: 0.30-0.75, p-value 0.002) and chocolate (OR: 0.544, 95\% CI: 0.34-0.88, p-value 0.017). No association between any other substance and ED was observed among males and females (Table 3).

\section{Gender-Based Differences in Behavioral Questions}

More females (5.5\%) than males (4.4\%) participated in vomiting to control their shape (p-value 0.042 ). Most of the vomiting occurred once per month $(22.5 \%)$. More males $(46.9 \%)$ than females $(36.9 \%)$ ever exercised more than 60 minutes every day to control their weight ( $\mathrm{p}$-value $<0.001)$. More males (18.5\%) than females (13.6\%) lost 20 pounds in the last six months (p-value 0.031). More females $(7.8 \%)$ than males $(4.2 \%)$ were treated for an ED (p-value 0.02) (Table 4).

Binary Logistic Regression Analysis of the Association Between EAT-26 and Substance Use and Other Factors

Based on EAT-26, the binary logistic regression revealed females were 2.93 times more likely to have ED than 
Table I Sociodemographic and Lifestyle Characteristics of the Students $(n=1047)$

\begin{tabular}{|c|c|c|c|c|}
\hline & & Males n (\%) & Females n (\%) & Total n (\%) \\
\hline \multirow[t]{3}{*}{ Residency } & City & $188(46.4)$ & $340(53.0)$ & $528(50.4)$ \\
\hline & Village & $20 I(49.6)$ & $293(45.6)$ & $494(47.2)$ \\
\hline & Refugee camp & $16(4.0)$ & $9(1.4)$ & $25(2.4)$ \\
\hline \multirow[t]{2}{*}{ Working status } & Working student & $96(23.7)$ & $33(5.1)$ & $129(12.3)$ \\
\hline & Not working student & $309(76.3)$ & $609(94.9)$ & $918(87.7)$ \\
\hline \multirow[t]{2}{*}{ Marital status } & Single & $402(99.1)$ & $626(97.5)$ & $1028(98.2)$ \\
\hline & Non-single & $3(0.7)$ & $16(2.5)$ & $19(1.8)$ \\
\hline \multirow[t]{9}{*}{ Substance practice } & Cigarette smoking & 159(39.3) & $25(3.9)$ & $184(17.6)$ \\
\hline & Water-pipe smoking & $176(43.5)$ & $139(21.7)$ & $315(30.1)$ \\
\hline & Electrical cigarette smoking & $36(8.9)$ & $13(2.0)$ & $49(4.7)$ \\
\hline & Energy drinks & $242(59.8)$ & 191 (29.8) & $433(4 I .4)$ \\
\hline & Coffee & $347(85.7)$ & $550(85.7)$ & $897(85.7)$ \\
\hline & Tea & $339(83.7)$ & $545(84.9)$ & $884(84.4)$ \\
\hline & Chocolate & $348(85.9)$ & $621(96.7)$ & $969(92.6)$ \\
\hline & Cigarettes and water-pipe (dual use) & $91(22.5)$ & $16(2.5)$ & $107(10.2)$ \\
\hline & Cigarettes or water-pipe (one of them) & $148(26.5)$ & $127(19.8)$ & $275(26.3)$ \\
\hline \multirow[t]{3}{*}{ Academic field } & Medicine & $122(30.5)$ & $119(18.7)$ & $24 I(23.2)$ \\
\hline & Health sciences & $123(30.8)$ & $209(32.8)$ & $332(32.0)$ \\
\hline & Non-medical fields & $155(38.8)$ & $310(48.6)$ & $465(44.8)$ \\
\hline \multirow[t]{5}{*}{ Weight category } & Underweight & $22(5.5)$ & $81(12.6)$ & $103(9.9)$ \\
\hline & Normal weight & $208(51.6)$ & $44 I(68.7)$ & $649(62.1)$ \\
\hline & Overweight & $120(29.8)$ & $94(14.6)$ & $214(20.5)$ \\
\hline & Obese & $42(10.4)$ & $22(3.4)$ & $64(6.1)$ \\
\hline & Over obese & $\mathrm{II}(2.7)$ & $4(0.6)$ & $15(1.4)$ \\
\hline \multirow[t]{2}{*}{ Body mass index } & $<25$ & $230(57.1)$ & $522(81.3)$ & $752(72.0)$ \\
\hline & $\geq 25$ & $173(42.9)$ & $120(18.7)$ & $293(28.0)$ \\
\hline
\end{tabular}

males ( $p$-value $<0.001$ ). Moreover, water-pipe smokers were more likely to have ED than none smokers (OR: 1.471, p-value 0.047), while coffee (OR: 0.581, p-value 0.014 ) and chocolate consumers were less likely to have ED than none consumers (OR: 0.530, p-value 0.041). No other associations were observed between EAT-26 and other substance use ( $\mathrm{p}$-value $\geq 0.05$ ). Compared to obese students, underweight students (OR: 0.103, p-value $<0.001$ ), normal weight students (OR: 0.186, p-value $<0.001$ ), and overweight students (OR: 0.463, p-value 0.007) were less likely to have EAT-26. The curricular study year did not contribute to increasing ED among students. Work status, marital status, and academic fields were not associated with EAT-26 (p-value $\geq 0.05$ ) (Table 5).

\section{Discussion}

The results of this study have demonstrated that the total prevalence of eating disorders attitudes and behaviors in
Palestinian university students was high based on both the EAT-26 screening test $(21.2 \% ; 17.1 \%$ among males, and $23.8 \%$ among females) and SCOFF screening test (31.5\%; $24.0 \%$ among males and $36.3 \%$ among females). Based on EAT-26 scores, the prevalence of ED in the present study in females was within the same ranges obtained in previous national studies. ${ }^{10,38}$ The absence of previous local research on males was one of the most salient constraints in predicting a change in the prevalence of disordered eating attitudes and behaviors based on both the EAT-26 and SCOFF screening tests. There seems to be a high level of under recognized and undertreated disordered eating behaviors among students at ANNU, specifically males. Globally, these results were higher than the average pooled point prevalence of $\mathrm{ED}$, which was estimated at $5.7 \%$ in women and $2.2 \%$ in men, ${ }^{51}$ and results from other universities in various international countries. ${ }^{51-57}$ Data from systematic review reported that Palestine is within the same ranges $(2-54.8 \%)$ of disordered eating attitude for 
Table 2 The Prevalence of Eating Disorder Based on EAT-26 and SCOFF Screening Test According to Different Factors

\begin{tabular}{|c|c|c|c|c|}
\hline & $\begin{array}{c}\text { EAT-26 } \geq 20 \\
\text { Yes } n(\%)\end{array}$ & P-value & $\begin{array}{c}\text { SCOFF } \geq 2 \\
\text { Yes } n(\%)\end{array}$ & P-value \\
\hline $\begin{array}{l}\text { Gender } \\
\text { Males } \\
\text { Females }\end{array}$ & $\begin{array}{c}69(17.1) \\
152(23.8)\end{array}$ & 0.010 & $\begin{array}{c}97(24.0) \\
232(36.3)\end{array}$ & $<0.001$ \\
\hline $\begin{array}{l}\text { Residency } \\
\text { City } \\
\text { Village } \\
\text { Camp }\end{array}$ & $\begin{array}{c}118(22.4) \\
99(20.1) \\
4(16.0)\end{array}$ & 0.576 & $\begin{array}{c}172(37.2) \\
152(30.8) \\
5(20.0)\end{array}$ & 0.367 \\
\hline $\begin{array}{l}\text { Marital Status } \\
\text { Single } \\
\text { Others }\end{array}$ & $\begin{array}{c}217(2 \mid .2) \\
4(2 \mid . I)\end{array}$ & 0.625 & $\begin{array}{c}323(31.5) \\
6(31.6)\end{array}$ & 0.993 \\
\hline $\begin{array}{l}\text { Study year } \\
\qquad \begin{array}{l}\text { Ist } \\
\text { 2nd } \\
\text { 3rd } \\
\text { 4th } \\
\text { 5th } \\
\text { 6th }\end{array}\end{array}$ & $\begin{array}{l}49(24.4) \\
62(22.5) \\
39(20.5) \\
37(21.3) \\
20(16.5) \\
13(16.0)\end{array}$ & 0.488 & $\begin{array}{l}73(36.3) \\
92(33.5) \\
64(33.5) \\
47(27.2) \\
34(27.9) \\
18(22.2)\end{array}$ & 0.131 \\
\hline $\begin{array}{l}\text { Academic field } \\
\text { Clinical } \\
\text { medicine } \\
\text { Preclinical } \\
\text { medicine } \\
\text { Other health } \\
\text { Sciences } \\
\text { Non-medical } \\
\text { fields }\end{array}$ & $\begin{array}{l}\text { II(II.I) } \\
32(22.5) \\
78(23.7) \\
99(20.9)\end{array}$ & 0.059 & $\begin{array}{l}10(10.1) \\
44(30.8) \\
116(35.4) \\
158(33.3)\end{array}$ & $<0.001$ \\
\hline $\begin{array}{l}\text { Substance in } \\
\text { use } \\
\text { Cigarettes } \\
\text { and water-pipe } \\
\text { Cigarettes } \\
\text { or water-pipe }\end{array}$ & $\begin{array}{l}28(25.2) \\
58(21.6)\end{array}$ & 0.497 & $\begin{array}{l}34(30.4) \\
91(33.8)\end{array}$ & 0.628 \\
\hline $\begin{array}{l}\text { Energy drinks } \\
\text { Yes } \\
\text { No }\end{array}$ & $\begin{array}{c}93(21.6) \\
128(20.9)\end{array}$ & 0.786 & $\begin{array}{l}146(33.8) \\
183(29.9)\end{array}$ & 0.177 \\
\hline $\begin{array}{l}\text { Cigarettes } \\
\text { Yes } \\
\text { No }\end{array}$ & $\begin{array}{c}37(20.2) \\
\mid 84(2 \mid .4)\end{array}$ & 0.729 & $\begin{array}{c}50(27.2) \\
582(32.4)\end{array}$ & 0.166 \\
\hline $\begin{array}{l}\text { Water-pipe } \\
\text { Yes } \\
\text { No }\end{array}$ & $\begin{array}{c}78(35.3) \\
143(64.7)\end{array}$ & 0.052 & $\begin{array}{l}109(33.1) \\
220(66.9)\end{array}$ & 0.141 \\
\hline $\begin{array}{l}\text { Tea } \\
\text { Yes } \\
\text { No }\end{array}$ & $\begin{array}{c}179(81.0) \\
42(19.0)\end{array}$ & 0.118 & $\begin{array}{c}269(30.5) \\
60(36.8)\end{array}$ & 0.111 \\
\hline
\end{tabular}

(Continued)
Table 2 (Continued).

\begin{tabular}{|c|c|c|c|c|}
\hline & $\begin{array}{c}\text { EAT-26 } \geq 20 \\
\text { Yes } n(\%)\end{array}$ & P-value & $\begin{array}{l}\text { SCOFF } \geq 2 \\
\text { Yes n(\%) }\end{array}$ & P-value \\
\hline \multicolumn{5}{|l|}{$\begin{array}{l}\text { Electrical } \\
\text { cigarettes }\end{array}$} \\
\hline Yes & II(22.4) & 0.822 & $17(34.7)$ & 0.620 \\
\hline No & $210(21.1)$ & & $3 \mid 2(3 \mid .3)$ & \\
\hline \multicolumn{5}{|l|}{ Coffee } \\
\hline Yes & $178(19.9)$ & 0.015 & $297(33.2)$ & 0.004 \\
\hline No & $43(28.7)$ & & $32(21.3)$ & \\
\hline \multicolumn{5}{|l|}{ Chocolate } \\
\hline Yes & $198(20.5)$ & 0.062 & $31 \mathrm{I}(32.2)$ & 0.097 \\
\hline No & $23(29.5)$ & & $18(23.1)$ & \\
\hline \multicolumn{5}{|l|}{$\begin{array}{l}\text { Weight } \\
\text { cataogory }\end{array}$} \\
\hline Underweight & $15(10.9)$ & $<0.001$ & $22(16.2)$ & $<0.001$ \\
\hline Normal & $106(17.3)$ & & $180(29.3)$ & \\
\hline \multicolumn{5}{|l|}{ weight } \\
\hline Overweight & $64(30.0)$ & & $92(43.2)$ & \\
\hline Obese & $36(45.6)$ & & $34(42.5)$ & \\
\hline
\end{tabular}

individuals in Arabic countries. ${ }^{58}$ Young adults in the Arab world, especially university students, tend to be in perfect shape to impress others. ${ }^{58-60}$ They have increased prevalence of eating disorders comparing to western and East Asian countries due to new western culture affecting our culture and the tendency and wellness to have unrealistic thin and muscular beauty ideals. ${ }^{59,60}$ This ultimately leads to increased incidence of psychological distress and eating disorders among them. ${ }^{11,12}$ The present study's overall prevalence was lower than the prevalence obtained in other Arab countries except in Lebanon and Sudan, which were close to our estimate. ${ }^{61,62}$ These results indicate that ED among Palestinian university students is more common than their international counterparts but compatible with some regional results. Therefore, there is a strong need to increase awareness and detection of ED among Palestinian university students.

Like other studies, females were significantly more likely to be identified as at risk of ED than males (p-value <0.001). These results (female prominence) were consistent with some other Arab and global countries $^{2,56,57,62-65}$ except in Egypt where no gender difference was observed, ${ }^{66}$ and in the United Arab of Emirates, Pakistan, China, and among Filipino students, 
Table 3 Univariate Analysis of the Association Between Eating Disorders Based on EAT-26 and Substances Used by the Students

\begin{tabular}{|c|c|c|c|c|c|c|}
\hline \multirow[t]{2}{*}{ Males } & & \multicolumn{2}{|c|}{ EAT-26 } & \multirow[b]{3}{*}{ OR } & \multirow[b]{3}{*}{$95 \%(\mathrm{Cl})$} & \multirow[b]{3}{*}{ P-value } \\
\hline & & Yes & NO & & & \\
\hline Substance in use & & $n(\%)$ & $n(\%)$ & & & \\
\hline Cigarette & $\begin{array}{l}\text { Yes } \\
\text { No }\end{array}$ & $\begin{array}{l}29(42.0) \\
40(58.0)\end{array}$ & $\begin{array}{l}129(38.5) \\
206(61.5)\end{array}$ & 1.129 & $0.73-1.74$ & 0.585 \\
\hline Water-pipe & $\begin{array}{l}\text { Yes } \\
\text { No }\end{array}$ & $\begin{array}{l}39(56.5) \\
30(43.5)\end{array}$ & $\begin{array}{l}136(40.6) \\
199(59.4)\end{array}$ & 1.701 & I.10-2.62 & 0.015 \\
\hline Electrical cigarette & $\begin{array}{l}\text { Yes } \\
\text { No }\end{array}$ & $\begin{array}{c}7(10.1) \\
62(89.9)\end{array}$ & $\begin{array}{c}29(8.7) \\
306(91.3)\end{array}$ & 1.154 & $0.57-2.23$ & 0.693 \\
\hline Energy drinks & $\begin{array}{l}\text { Yes } \\
\text { No }\end{array}$ & $\begin{array}{l}45(65.2) \\
24(34.8)\end{array}$ & $\begin{array}{l}196(58.5) \\
139(4 \mid .5)\end{array}$ & 1.268 & $0.81-1.99$ & 0.301 \\
\hline Coffee & $\begin{array}{l}\text { Yes } \\
\text { No }\end{array}$ & $\begin{array}{l}5 \mid(73.9) \\
18(26.1)\end{array}$ & $\begin{array}{c}295(88.1) \\
40(11.9)\end{array}$ & 0.475 & $0.30-0.75$ & 0.002 \\
\hline Tea & $\begin{array}{l}\text { Yes } \\
\text { No }\end{array}$ & $\begin{array}{l}55(79.7) \\
14(20.3)\end{array}$ & $\begin{array}{c}283(84.5) \\
52(15.5)\end{array}$ & 0.767 & $0.45-1.30$ & 0.329 \\
\hline Chocolate & $\begin{array}{l}\text { Yes } \\
\text { No }\end{array}$ & $\begin{array}{l}53(76.8) \\
16(23.2)\end{array}$ & $\begin{array}{c}294(87.8) \\
4 I(I 2.2)\end{array}$ & 0.544 & $0.34-0.88$ & 0.017 \\
\hline \multicolumn{7}{|l|}{ Females } \\
\hline Cigarette & $\begin{array}{l}\text { Yes } \\
\text { No }\end{array}$ & $\begin{array}{c}8(5.3) \\
\mid 44(94.7)\end{array}$ & $\begin{array}{c}I 7(3.5) \\
47 \mid(96.5)\end{array}$ & 1.37 & $0.76-2.46$ & 0.323 \\
\hline Water-pipe & $\begin{array}{l}\text { Yes } \\
\text { No }\end{array}$ & $\begin{array}{c}39(25.7) \\
113(74.3)\end{array}$ & $\begin{array}{c}99(20.3) \\
389(79.7)\end{array}$ & 1.255 & $0.92-|.7|$ & 0.160 \\
\hline Electrical cigarette & $\begin{array}{l}\text { Yes } \\
\text { No }\end{array}$ & $\begin{array}{c}4(2.6) \\
148(97.4)\end{array}$ & $\begin{array}{c}9(1.8) \\
479(98.2)\end{array}$ & 1.304 & $0.57-2.98$ & 0.519 \\
\hline Energy drinks & $\begin{array}{l}\text { Yes } \\
\text { No }\end{array}$ & $\begin{array}{c}48(31.6) \\
104(68.4)\end{array}$ & $\begin{array}{l}142(29.1) \\
346(70.9)\end{array}$ & 1.093 & $0.8 \mathrm{I}-1.47$ & 0.559 \\
\hline Coffee & $\begin{array}{l}\text { Yes } \\
\text { No }\end{array}$ & $\begin{array}{l}127(83.6) \\
25(16.4)\end{array}$ & $\begin{array}{c}42 I(86.3) \\
67(13.7)\end{array}$ & 0.853 & $0.59-1.23$ & 0.404 \\
\hline Tea & $\begin{array}{l}\text { Yes } \\
\text { No }\end{array}$ & $\begin{array}{l}124(81.6) \\
28(18.4)\end{array}$ & $\begin{array}{c}419(85.9) \\
69(14.1)\end{array}$ & 0.791 & $0.56-1.12$ & 0.199 \\
\hline Chocolate & $\begin{array}{l}\text { Yes } \\
\text { No }\end{array}$ & $\begin{array}{c}145(95.4) \\
7(4.6)\end{array}$ & $\begin{array}{c}474(97.1) \\
14(2.9)\end{array}$ & 0.703 & $0.38-1.31$ & 0.294 \\
\hline
\end{tabular}

Abbreviations: OR, odds ratio; $\mathrm{Cl}$, confidence interval.

where male predominance prevalence was observed. ${ }^{57,67-70}$ The female-to-male ratio of ED prevalence in this study was approximately 1.5 to 1 for both scale tests, which is noticeably lower than the global female to male ED prevalence (2.6 to 1$){ }^{51}$ This tighter gap in gender ratio among the university students implies that although Palestinian female students are at high risk for ED, their male counterparts are not much spared when compared to the global population. One explanation of this narrow gap could be that males were more likely to be overweight and obese $(42.9 \%)$ in this study than females (18.7\%). Similar findings have been reported in other studies. $^{71-74}$ This could be highly attributed to the fact that people with higher BMI are less satisfied with their body image, feeling low self-esteem, and desire to lose weight, which puts them at high risk of risky eating 
Table 4 Gender-Based Differences in Eating Behaviors Among Students

\begin{tabular}{|c|c|c|c|c|c|c|c|c|}
\hline \multirow[b]{2}{*}{ Question } & \multicolumn{7}{|c|}{ Behavior Frequency $n(\%)$} & \multirow[t]{2}{*}{ P-value } \\
\hline & Gender & Never & $\begin{array}{l}\text { Once } \\
\text { a Month }\end{array}$ & $\begin{array}{l}\text { 2-3 } \\
\text { Times/ } \\
\text { Month }\end{array}$ & $\begin{array}{l}\text { Once } \\
\text { a Week }\end{array}$ & $\begin{array}{l}\text { 2-6 } \\
\text { Times } \\
\text { Weekly }\end{array}$ & Daily & \\
\hline \multirow{2}{*}{$\begin{array}{l}\text { Ever gone on eating binges where you } \\
\text { feel that you may not be able to stop }\end{array}$} & Male & $253(62.5)$ & $91(22.5)$ & $26(6.4)$ & $17(4.2)$ & $8(2.0)$ & $10(2.5)$ & \multirow[t]{2}{*}{0.661} \\
\hline & Female & $386(60.1)$ & $50(23.4)$ & $50(7.8)$ & $29(4.5)$ & $18(2.8)$ & $9(1.4)$ & \\
\hline \multirow{2}{*}{$\begin{array}{l}\text { Ever made yourself sick (vomited) to } \\
\text { control your weight or shape? }\end{array}$} & Male & $387(95.6)$ & $5(1.2)$ & $8(2.0)$ & $3(0.7)$ & $2(0.5)$ & $0(0.0)$ & \multirow[t]{2}{*}{0.042} \\
\hline & Female & $607(94.5)$ & $21(3.3)$ & $4(0.6)$ & $8(1.2)$ & $\mathrm{I}(0.2)$ & $\mathrm{I}(0.2)$ & \\
\hline \multirow{2}{*}{$\begin{array}{l}\text { Ever used laxatives, diet pills or diuretics } \\
\text { (water pills) to control your weight or } \\
\text { shape? }\end{array}$} & Male & $385(95.1)$ & $7(1.7)$ & $2(0.5)$ & $5(1.2)$ & $2(0.5)$ & $4(1.0)$ & \multirow[t]{2}{*}{0.751} \\
\hline & Female & $6 \mid 4(95.6)$ & $\mathrm{II}(\mathrm{I} .7)$ & $6(0.9)$ & $3(0.5)$ & $2(0.3)$ & $6(0.9)$ & \\
\hline \multirow{3}{*}{$\begin{array}{l}\text { Ever exercised more than } 60 \text { minutes } \\
\text { a day to lose or to control your weight? }\end{array}$} & Male & $215(53.1)$ & $52(12.8)$ & $25(6.2)$ & $33(8.1)$ & $46(I I .4)$ & $34(8.4)$ & \multirow[t]{2}{*}{$<0.001$} \\
\hline & Female & $405(63.1)$ & $78(21.1)$ & $53(8.3)$ & $38(5.9)$ & $4 I(6.4)$ & $27(4.2)$ & \\
\hline & & No & Yes & & & & & \\
\hline \multirow{3}{*}{$\begin{array}{l}\text { Have Lost } 20 \text { pounds or more in the past } \\
6 \text { months? }\end{array}$} & Male & $330(81.5)$ & $75(18.5)$ & & & & & \multirow[t]{3}{*}{0.031} \\
\hline & Female & $554(86.4)$ & $87(13.6)$ & & & & & \\
\hline & Total & $884(84.5)$ & $162(15.5)$ & & & & & \\
\hline \multirow{3}{*}{$\begin{array}{l}\text { Have you ever been treated for an eating } \\
\text { disorder? }\end{array}$} & Male & $388(95.8)$ & $17(4.2)$ & & & & & \multirow[t]{3}{*}{0.020} \\
\hline & Female & $591(92.2)$ & $50(7.8)$ & & & & & \\
\hline & Total & $979(93.6)$ & $67(6.4)$ & & & & & \\
\hline
\end{tabular}

Notes: The EAT-26 has been reproduced with permission. Garner et al (1982). The Eating Attitudes Test: Psychometric features and clinical correlates. Psychological Medicine, 12, 87I-878. ${ }^{45}$ www.EAT-26.com.

behaviors. ${ }^{54}$ In agreement with other studies, females had a higher rate of purging behavior like vomiting to lose weight, while exercising for $60 \mathrm{~min}$ or more was more common among males than females. ${ }^{75}$ This could be another explanation for the high prevalence of ED among males in this study. These results could also indicate that young Palestinian males are more likely to develop diseases associated with obesity, such as metabolic diseases and heart diseases. ${ }^{76}$

Since no studies were conducted previously to detect this population at risk, the narrow gender gap in this study emphasizes the need for further studies to investigate the prevalence and the risk factors associated with increased risk of ED in males in the general Palestinian population. Due to societal stigma and lack of awareness, it is plausible that ED among Palestinian males may go unrecognized and undertreated. This delay in diagnosis could devastate psychiatric morbidity, leading to life-threatening conditions. ${ }^{44}$
Medical students have higher rates of psychiatric disorders given the chronic stress and risk of burnout they are subjected to, particularly concerning eating disorders. ${ }^{15}$ Similar to the results from other Arab countries, ${ }^{62,66}$ the prevalence of ED based on EAT-26 among medical students in this study was higher than the global average pooled point prevalence, which was estimated at $10.4 \%$, and higher than the reported prevalence in different countries among medical students. ${ }^{16,65,77,78}$ As for universityrelated characteristics among our participants, students at the medical sciences faculties had similar odds of being ED than those studying at non-medicinal sciences faculties. However, clinical medicine students had the lowest prevalence among all other students. One possible explanation is that medical students have more knowledge about ED than students do in other academic specialties. Thus, this higher ED awareness could have decreased its prevalence among them. However, the high prevalence of ED 
Table 5 Binary Logistic Regression of the Association Between Eating Disorders Based on EAT-26 Substances in Use

\begin{tabular}{|c|c|c|c|c|}
\hline EAT-26 Yes ${ }^{a}$ & & Odds Ratio & $95 \%(\mathrm{Cl})$ & P-value \\
\hline Study year & & 0.940 & $0.823-1.073$ & $0.36 \mathrm{I}$ \\
\hline Gender & $\begin{array}{l}\text { Male } \\
\text { Female }\end{array}$ & $\begin{array}{l}0.34 \mathrm{I} \\
\text { Reference group }\end{array}$ & $0.221-0.526$ & $<0.001$ \\
\hline Work status & $\begin{array}{l}\text { Yes } \\
\text { No }\end{array}$ & $\begin{array}{l}1.372 \\
\text { Reference group }\end{array}$ & $0.832-2.262$ & 0.215 \\
\hline Marital Status & $\begin{array}{l}\text { Single } \\
\text { Not single }\end{array}$ & $\begin{array}{l}0.722 \\
\text { Reference group }\end{array}$ & $0.227-2.296$ & 0.582 \\
\hline Academic field & $\begin{array}{l}\text { Clinical medicine } \\
\text { Preclinical medicine } \\
\text { Other health Sciences } \\
\text { Non-medical fields }\end{array}$ & $\begin{array}{l}0.590 \\
1.109 \\
1.107 \\
\text { Reference group }\end{array}$ & $\begin{array}{l}0.276-1.262 \\
0.668-1.840 \\
0.757-1.618\end{array}$ & $\begin{array}{l}0.173 \\
0.690 \\
0.602\end{array}$ \\
\hline Weight category & $\begin{array}{l}\text { Underweight } \\
\text { Normal } \\
\text { Overweight } \\
\text { Obese }\end{array}$ & $\begin{array}{l}0.103 \\
0.186 \\
0.463 \\
\text { Reference group }\end{array}$ & $\begin{array}{l}0.049-0.215 \\
0.108-0.318 \\
0.264-0.813\end{array}$ & $\begin{array}{l}<0.001 \\
<0.001 \\
0.007\end{array}$ \\
\hline Cigarette smoking ${ }^{b}$ & Yes & 1.122 & $0.67 \mid-1.877$ & 0.660 \\
\hline Water-pipe smoking ${ }^{b}$ & Yes & I.47I & $1.005-2.154$ & 0.047 \\
\hline Electrical cigarette ${ }^{b}$ & Yes & 0.967 & $0.447-2.092$ & 0.932 \\
\hline Energy drinks ${ }^{b}$ & Yes & 1.120 & $0.777-1.613$ & 0.544 \\
\hline Coffee ${ }^{b}$ & Yes & 0.581 & $0.378-0.895$ & 0.014 \\
\hline Tea ${ }^{b}$ & Yes & 0.988 & $0.635-1.535$ & 0.956 \\
\hline Chocolate $^{b}$ & Yes & 0.530 & $0.288-0.976$ & 0.041 \\
\hline
\end{tabular}

Notes: ${ }^{a}$ The reference group is EAT-26 No, ${ }^{\text {b}}$ The reference group substance use NO.

Abbreviations: $\mathrm{OR}$, odds ratio; $\mathrm{Cl}$, confidence Interval.

either among both genders and medical and non-medical students calls for the need to create awareness-raising campaigns about ED targeting university students and create effective screening protocols easy-to-access psychological consultation for university students.

Smoking could be used as a weight-control strategy especially by women affected by ED. ${ }^{79-81}$ It was reported that a higher prevalence of smoking in a clinical sample of individuals with bulimia nervosa than in individuals with anorexia nervosa, whereas another study did not detect ED subtype differences in smoking prevalence. ${ }^{82,83}$ Although our results suggest that ED rate is not associated with higher cigarette smoking rates or energy drinks consumption, a positive relation between EAT-26 and water-pipe smoking among males was found (OR: 1.47, p-value 0.047), indicating that water-pipe smoking among male university students could be associated with unhealthy food behaviors for weight loss. ${ }^{19,21-23,84}$ The prevalence and pattern of cigarette smoking and energy drinks used in ED individuals were not significantly different from those without ED ( $p$-value $>0.05$ ). The results have shown that students' smoking is motivated more by desires for fun, peer pressure, nicotine dependence, and to less extent for weight control. It could be that the perceived benefits of nicotine for temporary stress reduction may outweigh the benefit for weight-control and any concerns about the long-term harms of cigarette smoking among students. Overall, routine smoking assessment and smoking cessation treatment is warranted for those with ED by health care providers. $^{82}$

This study revealed that male students who consumed coffee had a lower risk of having ED than non-consumers (OR: 0.475 , p-value 0.002). The relationship between coffee consumption with ED somehow remains vague and 
needs more analysis and more research on specific types of eating disorders and their relation to coffee consumption. ${ }^{25,85,86}$ Chocolate consumers were also at lower risk if ED than non-consumers (OR: 0.544, p-value 0.017). These results agree with the results of a previous study. ${ }^{87}$ It has been found that there is a relationship between guilt feeling when eating chocolate and developing abnormal eating behaviors; the high the guilt score, the more tendency to report symptoms such as binging and vomiting. ${ }^{88}$ Chocolate-induced guilt, emotional eating, and restrain were far more common in females than males, even if the consumption level is the same. ${ }^{87}$ Importantly, no significant association between any ED and coffee or chocolate consumption within females was found in the current study ( $p$-value $>0.05$ ). These gender differences emphasize the need for further studies to determine other risk factors and the directionality of such associations. These results will help the medical community better understand and better prevent ED in both males and females.

\section{Limitations and Strength}

This study has some limitations. The results were based on students' self-reported information, which may represent some bias, as students may not be accurate in reporting their behaviors. No inferences could be made regarding causality, as this is a cross-sectional study. The scales used in this study cannot provide a diagnosis, and the lack of mental health assessment by experts made our study valuable only as an initial screening method. Results should be interpreted with caution since no clinical diagnostic data were collected to validate ED screening and report specific types. Moreover, the study was conducted during the COVID-19 pandemic. In the setting of COVID-19 quarantine, the possible effect of COVID-19 on ED and its associations among students is unknown. Despite these limitations, this is the first study investigating and establishing gender-based differences in the prevalence and risk factors associated with the disordered eating behaviors and attitudes in males in Palestine. It is also the first one to assess the association between unhealthy eating attitudes and other highly prevalent factors among university students, including cigarette smoking, water-pipe, energy drinks, coffee, tea, and chocolate consumption.

\section{Conclusion}

Eating disorders in Palestinian male students is a neglected health issue. We aimed to investigate the prevalence and the association between ED and cognitive enhancers and psychostimulants use among An-Najah National University students. The results of this study showed an alarming prevalence of disordered eating attitudes in both genders and among those with higher BMI. ED in male students was an under-recognized and neglected health issue in previous national studies. The ED gender gap in university students was very narrow compared to international results, which prompts the need to focus on both genders in future ED studies instead of females. Our findings highlight water-pipe smoking as a significant health problem in males with $\mathrm{ED}$, which may require unique treatment and prevention strategies. Moreover, coffee and chocolate consumption were associated with a significantly decreased risk of ED among males only. Further investigation is required to address the direction of this relationship. Clinical medical students had the lowest prevalence of ED among university students. Based on these results, we recommend conducting further research to investigate the prevalence and the risk factors of eating disorders in the general population. More attention through university screening programs should be given to the university students, with equal focus on both genders, to reduce the risk of eating disorders and associated morbidity and mortality, and to increase awareness about smoking problems, cigarette, or water-pipe.

\section{Abbreviations}

ANNU, An-Najah National University; ANOVA, analysis of variances; CI, confidence interval; EAT-26, The Eating attitude test-26; ED, Eating disorders; SCOFF, Sick, Control, One, Fat and FOOD (SCOFF) screening tests; OR, odds ratio.

\section{Data Sharing Statement}

Most data generated or analyzed during this study are included in this manuscript. Other data that support the findings of this study and/or analyzed during the current study are available from the corresponding author on reasonable request.

\section{Informed Consent}

The Ethics Committee of the Institutional Review Board "IRB" approval at An-Najah National University (ANNU) was obtained to confirm that the study matches the ethical research protocols. All procedures followed were in accordance with the ethical standards of the responsible committee on human experimentation (institutional and 
national) and with the Helsinki Declaration of 1975, as revised in 2000. Informed consent was obtained from all participants for being included in the study. An anonymous questionnaire was used. Participants had the ability to assent to or dissent from participation. Investigators from both genders were chosen to conduct this research as taking some data such as weight and height could be embarrassing for the participants if the investigator was from the opposite gender. Participants were assured that all of the data collected would only be kept confidential and only accessible for the investigators and research purposes. The questionnaires were kept in a secure place, and serial numbers were used to refer to participants during analysis and presenting data. The EAT-26 has been reproduced with permission Garner et al (1982). ${ }^{45}$

\section{Acknowledgment}

The authors would like to thank the respondents for their participation in this study. The authors are very thankful to all those who facilitated the conduction of this study in ANNU; Ahmed Mousa, Abdulraziq Zarour, Mohammad Badwan, Yazan Al Habil, Lara Mohsin, Ali Shalabi, and Ansam Mousa.

\section{Author Contributions}

All authors contributed to data analysis, drafting or revising the article, have agreed on the journal to which the article will be submitted, gave final approval of the version to be published, and agree to be accountable for all aspects of the work.

\section{Funding}

There is no funding to report.

\section{Disclosure}

The authors declare that they have no conflicts of interest.

\section{References}

1. Klump KL, Bulik CM, Kaye WH, Treasure J, Tyson E. Academy for eating disorders position paper: eating disorders are serious mental illnesses. Int J Eat Disord. 2009;42(2):97-103. doi:10.1002/eat.20589

2. Memon AA, Adil SE, Siddiqui EU, Naeem SS, Ali SA, Mehmood K. Eating disorders in medical students of Karachi, Pakistan-A Cross-Sectional Study. BMC Res Notes. 2012;5(1):84. doi:10.1186/ 1756-0500-5-84

3. Hoek HW. Incidence, prevalence and mortality of anorexia nervosa and other eating disorders. Curr Opin Psychiatry. 2006;19(4):389-394. doi:10.1097/01.yco.0000228759.95237.78
4. Aoun A, Azzam J, Jabbour FE, et al. Validation of the Arabic version of the SCOFF questionnaire for the screening of eating disorders. East Mediterr Health J. 2015;21(5):326-331.

5. World Health Organization. Gender in Mental Health Research. 2004.

6. Tozzi F, Sullivan PF, Fear JL, McKenzie J, Bulik CM. Causes and recovery in anorexia nervosa: the patient's perspective. Int $J$ Eat Disord. 2003;33(2):143-154. doi:10.1002/eat.10120

7. Klump KL, Miller KB, Keel PK, McGue M, Iacono WG. Genetic and environmental influences on anorexia nervosa syndromes in a population-based twin sample. Psychol Med. 2001;31(4):737-740. doi:10.1017/S0033291701003725

8. Cooley E, Toray T. Disordered eating in college freshman women: a Prospective Study. J Am Coll Health. 2001;49(5):229-235. doi:10.1080/07448480109596308

9. Kaluski DN, Natamba BK, Goldsmith R, Shimony T, Berry EM. Determinants of disordered eating behaviors among Israeli adolescent girls. Eat Disord. 2008;16(2):146-159. doi:10.1080/ 10640260801887303

10. Saleh RN, Salameh RA, Yhya HH, Sweileh WM. Disordered eating attitudes in female students of An-Najah National University: a Cross-Sectional Study. J Eat Disord. 2018;6(1):16. doi:10.1186/ s40337-018-0204-4

11. Stice E, Maxfield J, Wells T. Adverse effects of social pressure to be thin on young women: an experimental investigation of the effects of "fat talk". Int J Eat Disord. 2003;34(1):108-117. doi:10.1002/ eat. 10171

12. Aparicio-Martinez P, Perea-Moreno AJ, Martinez-Jimenez MP, Redel-Macías MD, Pagliari C, Vaquero-Abellan M. Social media, thin-ideal, body dissatisfaction and disordered eating attitudes: an exploratory analysis. Int J Environ Res Public Health. 2019;16 (21):21. doi:10.3390/ijerph16214177

13. Kiss-Tóth E, Wasilewska M, Sopel O, et al. Eating disorder in university students: an International Multi-Institutional Study. Eur J Public Health. 2018;28(suppl_4). doi:10.1093/eurpub/cky214.010

14. Eisenberg D, Nicklett EJ, Roeder K, Kirz NE. Eating disorder symptoms among college students: prevalence, persistence, correlates, and treatment-seeking. $J$ Am Coll Health. 2011;59(8):700-707. doi:10.1080/07448481.2010.546461

15. Heinen I, Bullinger M, Kocalevent R-D. Perceived stress in first year medical students-associations with personal resources and emotional distress. BMC Med Educ. 2017;17(1):4. doi:10.1186/s12909-0160841-8

16. Jahrami H, Sater M, Abdulla A, AlAnsari A. Eating disorders risk among medical students: a global systematic review and meta-analysis. Eat Weight Disord. 2019;24(3):397-410. doi:10.1007/s40519-018-0516-Z

17. Hoerr SL, Bokram R, Lugo B, Bivins T, Keast DR. Risk for disordered eating relates to both gender and ethnicity for college students. J Am Coll Nutr. 2002;21(4):307-314. doi:10.1080/ 07315724.2002.10719228

18. Mahoney CR, Giles GE, Marriott BP, et al. Intake of caffeine from all sources and reasons for use by college students. Clin Nutr. 2019;38 (2):668-675. doi:10.1016/j.clnu.2018.04.004

19. Neumark-Sztainer D, Story M, French SA. Covariations of unhealthy weight loss behaviors and other high-risk behaviors among adolescents. Arch Pediatr Adolesc Med. 1996;150(3):304-308. doi:10.1001/archpedi.1996.02170280074014

20. Jo YH, Talmage DA, Role LW. Nicotinic receptor-mediated effects on appetite and food intake. J Neurobiol. 2002;53(4):618-632. doi:10.1002/neu.10147

21. Alomari MA, Al-Sheyab NA, Ward KD. Adolescent waterpipe use is associated with greater body weight: the Irbid-TRY. Subst Use Misuse. 2018;53(7):1194-1202. doi:10.1080/ 10826084.2017.1400065 
22. Ward KD, Ahn S, Mzayek F, et al. The relationship between waterpipe smoking and body weight: population-based findings from Syria. Nicotine Tob Res. 2015;17(1):34-40. doi:10.1093/ntr/ntu121

23. Berro J, Akel M, Hallit S. Relationships between inappropriate eating habits and problematic alcohol use, cigarette and waterpipe dependence among male adolescents in Lebanon. BMC Public Health. 2021;21(1):140. doi:10.1186/s12889-021-10184-2

24. Hart S, Abraham S, Luscombe G, Russell J. Fluid intake in patients with eating disorders. Int $J$ Eat Disord. 2005;38(1):55-59. doi:10.1002/eat.20155

25. Winston AP, Hardwick E, Jaberi N. Neuropsychiatric effects of caffeine. Adv Psychiatr Treat. 2018;11(6):432-439. doi:10.1192/ apt.11.6.432

26. Jeffers AJ, Vatalaro Hill KE, Benotsch EG. Energy drinks, weight loss, and disordered eating behaviors. J Am Coll Health. 2014;62 (5):336-342. doi:10.1080/07448481.2014.902838

27. Hetherington MM, Macdiarmid JI. Pleasure and excess: liking for and overconsumption of chocolate. Physiol Behav. 1995;57(1):27-35. doi:10.1016/0031-9384(94)00199-F

28. Tuomisto T, Hetherington MM, Morris MF, Tuomisto MT, Turjanmaa V, Lappalainen R. Psychological and physiological characteristics of sweet food "addiction". Int J Eat Disord. 1999;25 (2):169-175. doi:10.1002/(SICI)1098-108X(199903)25:2<169::AIDEAT6>3.0.CO;2-B

29. Massad SG, Shaheen M, Karam R, et al. Substance use among Palestinian youth in the West Bank, Palestine: a qualitative investigation. BMC Public Health. 2016;16(1):800. doi:10.1186/ s12889-016-3472-4

30. Musmar SG. Smoking habits and attitudes among university students in Palestine: a Cross-Sectional Study. East Mediterr Health J. 2012;18(5):454-460. doi:10.26719/2012.18.5.454

31. Zaher Nazzal MAA-H, Musmar S. Prevalence of water-pipe smoking and associated factors among university students in Palestine: a Cross Sectional Study. Palestinian Med Pharm J. 2020;5(2):106-107.

32. Damiri B, Sandouka HN, Janini EH, Yaish ON. Prevalence and associated factors of psychoactive substance use among university students in the West Bank, Palestine. Drugs. 2020;27(2):173-182.

33. Tucktuck M, Ghandour R, Abu-Rmeileh NME. Waterpipe and cigarette tobacco smoking among Palestinian University students: a Cross-Sectional Study. BMC Public Health. 2017;18(1):1. doi:10.1186/s12889-017-4524-0

34. Sabbah HA. Consumption patterns and side effects of energy drinks among university students in palestine: Cross-Sectional Study. MOJ Public Health. 2015;2(2):36-44. doi:10.15406/mojph.2015.02.00015

35. Zaher Nazzal MAA-H, Musmar S. Social norms and perception of waterpipe smoking among Palestinian university students. Palestinian Med Pharm J. 2020;5(1):25-34.

36. Damiri B, Salahat I, Aghbar M. Pattern of substance use among schoolchildren in Palestine: a Cross-Sectional Study. Egypt J Forensic Sci. 2018;8(1):59. doi:10.1186/s41935-018-0090-6

37. Zabadi HA, Musmar S, Hassouna A, Shtaiwi D. Cigarettes and water pipe smoking prevalence, knowledge, and attitudes among the Palestinian physicians in the West Bank. Tob Use Insights. 2018;11:1179173X18813369. doi:10.1177/1179173X18813369

38. Zaher N, Watan N, Beesan M. Body Perceptions and Weight Control Behaviors Among Palestinian University Female Students: A CrossSectional Study. 2020:1-15. Located at: Research Square.

39. Zaher N, Watan N, Beesan M. Research square. 2021.

40. Strother E, Lemberg R, Stanford SC, Turberville D. Eating disorders in men: underdiagnosed, undertreated, and misunderstood. Eat Disord. 2012;20(5):346-355. doi:10.1080/10640266.2012.715512

41. Sangha S, Oliffe JL, Kelly MT, McCuaig F. Eating disorders in males: how primary care providers can improve recognition, diagnosis, and treatment. Am J Mens Health. 2019;13 (3): 1557988319857424 . doi:10.1177/1557988319857424
42. Ibrahim AK, Kelly SJ, Adams CE, Glazebrook C. A systematic review of studies of depression prevalence in university students. $J \quad$ Psychiatr Res. 2013;47(3):391-400. doi:10.1016/j. jpsychires.2012.11.015

43. Freeman A, Mergl R, Kohls E, et al. A cross-national study on gender differences in suicide intent. BMC Psychiatry. 2017;17(1):234. doi:10.1186/s12888-017-1398-8

44. Sweeting H, Walker L, MacLean A, Patterson C, Räisänen U, Hunt K. Prevalence of eating disorders in males: a review of rates reported in academic research and UK mass media. Int $J$ Mens Health. 2015;14(2). doi:10.3149/jmh.1402.3186

45. Garner DM, Olmsted MP, Bohr Y, Garfinkel PE. The eating attitudes test: psychometric features and clinical correlates. Psychological medicine. 1982;12(4):871-878

46. Morgan JF, Reid F, Lacey JH. The SCOFF questionnaire: a new screening tool for eating disorders. West J Med. 2000;172 (3):164-165. doi:10.1136/ewjm.172.3.164

47. Morgan JF, Reid F, Lacey JH. The SCOFF questionnaire: assessment of a new screening tool for eating disorders. BMJ. 1999;319 (7223):1467-1468. doi:10.1136/bmj.319.7223.1467

48. Al-Subaie A, Al-Shammari S, Bamgboye E, Al-Sabhan K, AlShehri S, Bannah AR. Validity of the Arabic version of the eating attitude test. Int $J$ Eat Disord. 1996;20(3):321-324. doi:10.1002/ (SICI)1098-108X(199611)20:3<321::AID-EAT12>3.0.CO;2-2

49. Damiri B, Abualsoud MS, Samara AM, Salameh SK. Metabolic syndrome among overweight and obese adults in Palestinian refugee camps. Diabetol Metab Syndr. 2018;10(1):34. doi:10.1186/s13098018-0337-2

50. Metintaş S, Sariboyaci M, Nuhoğlu S, et al. Smoking patterns of university students in Eskişehir, Turkey. Public Health. 1998;112 (4):261-264. doi:10.1016/S0033-3506(98)00243-1

51. Galmiche M, Déchelotte P, Lambert G, Tavolacci MP. Prevalence of eating disorders over the 2000-2018 period: a systematic literature review. Am J Clin Nutr. 2019;109(5):1402-1413. doi:10.1093/ajen/nqy342

52. Zulfiqar A, Laghari Z, Warsi J, Memon M, Lashari K, Ali Muhammad S. Detection of eating disorders in university students using SCOFF questionniare. Int J Dev Res. 2014;4(8):1634-1637.

53. Tavolacci MP, Grigioni S, Richard L, Meyrignac G, Déchelotte P, Ladner J. Eating disorders and associated health risks among university students. J Nutr Educ Behav. 2015;47(5):412-420.e411. doi:10.1016/j.jneb.2015.06.009

54. Balhara YPS, Mathur S, Kataria DK. Body shape and eating attitudes among female nursing students in India. East Asian Arch Psychiatry. 2012;22(2):70-74.

55. Costa LC, Vasconcelos FA, Peres KG. Influence of biological, social and psychological factors on abnormal eating attitudes among female university students in Brazil. J Health Popul Nutr. 2010;28 (2):173-181.

56. Reyes-Rodríguez ML, Franko DL, Matos-Lamourt A, et al. Eating disorder symptomatology: prevalence among Latino college freshmen students. J Clin Psychol. 2010;66(6):666-679. doi:10.1002/ jclp. 20684

57. Madanat HN, Hawks SR, Novilla ML. A comparison of disordered eating attitudes and behaviors among Filipino and American college students. Eat Weight Disord. 2006;11(3):133-138. doi:10.1007/ BF03327558

58. Melisse B, de Beurs E, van Furth EF. Eating disorders in the Arab world: a literature review. J Eat Disord. 2020;8(1):59. doi:10.1186/ s40337-020-00336-x

59. Thomas J, Al Marzooqi FH, Tahboub-Schulte S, Furber SW. Changing physical appearance preferences in the United Arab Emirates. Ment Health Relig Cult. 2014;17(6):594-600. doi:10.1080/13674676.2013.871242

60. Ford KA, Dolan BM, Evans C. Cultural factors in the eating disorders: a study of body shape preferences of Arab students. J Psychosom Res. 1990;34(5):501-507. doi:10.1016/0022-3999(90)90024-X 
61. Bizri M, Geagea L, Kobeissy F, Talih F. Prevalence of eating disorders among medical students in a lebanese medical school: a Cross-Sectional Study. Neuropsychiatr Dis Treat. 2020; 16:1879-1887.

62. Fath Al Alim MA, Noon HA, Abdelghani A. Eating disorders symptoms among a university students: an Exploratory Study. Sudan Med J. 2012;48(3):186-193.

63. Musaiger AO, Al-Kandari FI, Al-Mannai M, et al. Disordered eating attitudes among university students in Kuwait: the role of gender and obesity. Int $J$ Prev Med. 2016;7(1):67. doi:10.4103/20087802.180413

64. Pengpid S, Peltzer K, Ahsan GU. Risk of eating disorders among university students in Bangladesh. Int J Adolesc Med Health. 2015;27 (1):93-100. doi:10.1515/ijamh-2014-0013

65. Naeimi AF, Haghighian HK, Gargari BP, Alizadeh M, Rouzitalab T. Eating disorders risk and its relation to self-esteem and body image in Iranian university students of medical sciences. Eat Weight Disord. 2016;21(4):597-605. doi:10.1007/s40519-016-0283-7

66. Shehata WM. Eating disorder risk among medical students at Tanta University, Egypt. Egypt J Community Med. 2020;38(4):17-23.

67. Musaiger AO, Al-Mannai M, Al-Lalla O. Risk of disordered eating attitudes among male adolescents in five Emirates of the United Arab Emirates. Int $J$ Eat Disord. 2014;47(8):898-900. doi:10.1002/ eat. 22256

68. Thomas J, Khan S, Abdulrahman AA. Eating attitudes and body image concerns among female university students in the United Arab Emirates. Appetite. 2010;54(3):595-598. doi:10.1016/j. appet.2010.02.008

69. Babar N, Alam M, Ali SS, et al. Anorexic behaviour and attitudes among female medical and nursing students at a private university hospital. J Pak Med Assoc. 2002;52(6):272-276.

70. Yu J, Lu M, Tian L, et al. Prevalence of disordered eating attitudes among university students in Wuhu, China. Nutr Hosp. 2015;32 (4):1752-1757. doi:10.3305/nh.2015.32.4.9187

71. Burnette CB, Simpson CC, Mazzeo SE. Relation of BMI and weight suppression to eating pathology in undergraduates. Eat Behav 2018;30:16-21. doi:10.1016/j.eatbeh.2018.05.003

72. Alkazemi D, Zafar TA, Ebrahim M, Kubow S. Distorted weight perception correlates with disordered eating attitudes in Kuwaiti college women. Int J Eat Disord. 2018;51(5):449-458. doi:10.1002/ eat. 22852

73. Yang SJ, Kim JM, Yoon JS. Disturbed eating attitudes and behaviors in South Korean boys and girls: a School-Based Cross-Sectional Study. Yonsei Med J. 2010;51(3):302-309. doi:10.3349/ ymj.2010.51.3.302

74. Desai MN, Miller WC, Staples B, Bravender T. Risk fctors associated with overweight and obesity in college students. J Am Coll Health. 2008;57(1):109-114. doi:10.3200/JACH.57.1.109-114
75. Mangweth-Matzek B, Kummer KK, Pope HG. Eating disorder symptoms in middle-aged and older men. Int J Eat Disord. 2016;49 (10):953-957. doi:10.1002/eat.22550

76. Abdelaal M, le Roux CW, Docherty NG. Morbidity and mortality associated with obesity. Ann Transl Med. 2017;5(7):161. doi:10.21037/atm.2017.03.107

77. Liao Y, Knoesen NP, Castle DJ, et al. Symptoms of disordered eating, body shape, and mood concerns in male and female Chinese medical students. Compr Psychiatry. 2010;51(5):516-523. doi:10.1016/j. comppsych.2009.11.007

78. Balhara YP, Yadav T, Arya K, Mathur S, Kataria DK. A cross-sectional study of body shape and eating attitude among Indian female healthcare students. Int J Psychiatry Med. 2012;43 (4):309-323. doi:10.2190/PM.43.4.b

79. Haug NA, Heinberg LJ, Guarda AS. Cigarette smoking and its relationship to other substance use among eating disordered inpatients. Eat Weight Disord. 2001;6(3):130-139. doi:10.1007/ BF03339762

80. von Ranson KM, McGue M, Iacono WG. Disordered eating and substance use in an epidemiological sample: II. Associations within families. Psychol Addict Behav. 2003;17(3):193-201. doi:10.1037/ 0893-164X.17.3.193

81. Fairweather-Schmidt K, Wade TD. The relationship between disordered eating and cigarette smoking among adult female twins. Int J Eat Disord. 2015;48(6):708-714. doi:10.1002/eat.22363

82. Solmi M, Veronese N, Sergi G, et al. The association between smoking prevalence and eating disorders: a systematic review and meta-analysis. Addiction. 2016;111(11):1914-1922. doi:10.1111/ add. 13457

83. Anzengruber D, Klump KL, Thornton L, et al. Smoking in eating disorders. Eat Behav. 2006;7(4):291-299. doi:10.1016/j. eatbeh.2006.06.005

84. Neergaard J, Singh P, Job J, Montgomery S. Waterpipe smoking and nicotine exposure: a review of the current evidence. Nicotine Tob Res. 2007;9(10):987-994. doi:10.1080/14622200701591591

85. Ciapparelli A, Paggini R, Carmassi C, et al. Patterns of caffeine consumption in psychiatric patients. An Italian Study. Eur Psychiatry. 2020;25(4):230-235. doi:10.1016/j.eurpsy.2009.02.010

86. Burgalassi A, Ramacciotti CE, Bianchi M, et al. Caffeine consumption among eating disorder patients: epidemiology, motivations, and potential of abuse. Eat Weight Disord. 2009;14(4):e212-e218. doi:10.1007/BF03325119

87. Hormes JM, Orloff NC, Timko CA. Chocolate craving and disordered eating. Appetite. 2014;83:185-193. doi:10.1016/j. appet.2014.08.018

88. Benton D, Greenfield K, Morgan M. The development of the attitudes to chocolate questionnaire. Pers Individ Dif. 1998;24 (4):513-520. doi:10.1016/S0191-8869(97)00215-8
Neuropsychiatric Disease and Treatment

\section{Publish your work in this journal}

Neuropsychiatric Disease and Treatment is an international, peerreviewed journal of clinical therapeutics and pharmacology focusing on concise rapid reporting of clinical or pre-clinical studies on a range of neuropsychiatric and neurological disorders. This journal is indexed on PubMed Central, the 'PsycINFO' database and CAS, and is the official journal of The International Neuropsychiatric Association (INA). The manuscript management system is completely online and includes a very quick and fair peer-review system, which is all easy to use. Visit http://www.dovepress.com/testimonials.php to read real quotes from published authors. 